\title{
Predictive Factors for Acute Urinary Retention After Transperineal Template-Guided Mapping Biopsy
}

\author{
Si Hyun Sung, Chung Un Lee, Jae Hoon Chung, Wan Song, Minyong Kang, Hyun Hwan Sung, \\ Byong Chang Jeong, Seong II Seo, Seong Soo Jeon, Hyun Moo Lee, Hwang Gyun Jeon
}

Department of Urology, Samsung Medical Center, Sungkyunkwan University School of Medicine, Seoul, Korea

\begin{abstract}
Purpose: We investigated the predictive factors for acute urinary retention (AUR) after transperineal template-guided mapping biopsy (TTMB).

Materials and Methods: We retrospectively reviewed the records of 459 patients who had undergone TTMB between May 2017 and July 2020. Overall complications after TTMB were analyzed and categorized according to the Clavien-Dindo classification. Factors that were likely to affect AUR were analyzed using a logistic regression model.

Results: Overall complications after TTMB were observed in 95 of the 459 patients (20.7\%), of which AUR was the most commonly reported $(17.4 \%, \mathrm{n}=80)$, followed by hematuria $(3.1 \%$, $\mathrm{n}=14$ ). Hematuria in one patient was categorized as Clavien-Dindo grade Illa. All remaining complications were Clavien-Dindo grade I. In the multivariate regression model, age $\geq 65$ (odds ratio, $2.44 ; 95 \%$ confidence interval $[\mathrm{Cl}], 1.42-4.17 ; \mathrm{p}=0.001$ ), prostate volume $\geq 30 \mathrm{~mL}$ (odds ratio, 3.72; $95 \% \mathrm{Cl}, 1.19-11.62 ; \mathrm{p}<0.02$ ), and number of biopsy cores $\geq 30$ (odds ratio, $2.89 ; 95 \% \mathrm{Cl}$, 1.29-6.43; $p=0.01$ ) were identified as the predictors for AUR after TTMB.

Conclusions: AUR is the most common complication after TTMB. Age $\geq 65$ years, prostate volume $\geq 30 \mathrm{~mL}$, and number of biopsy cores $\geq 30$ were significant predictors of AUR following TTMB.
\end{abstract}

Key Words: Prostate biopsy, Transperineal, Complication, Acute urinary retention, Predictor
Received April 22, 2021

Revised June 6, 2021

Accepted June 24, 2021

Corresponding author:

Hwang Gyun Jeon

Email: hwanggyun.jeon@samsung.com https://orcid.org/0000-0002-5613-8389

\section{INTRODUCTION}

Transrectal ultrasound-guided biopsy (TRUS$\mathrm{Bx}$ ) plays an important role in the detection of prostate cancer in patients with increased prostate-specific antigen (PSA) levels or palpable nodule on a digital rectal exam. ${ }^{1,2}$ However, several studies have reported the limitations of TRUS-Bx, such as difficult access for cancers located anteriorly or at the apex, high rate of complications such as infection, and procedural difficulty in patients with rectal problems. ${ }^{3-8}$

Transperineal template mapping biopsy (TTMB) overcomes the shortcomings of the conventional TRUS- $\mathrm{Bx}$, and can provide access to the entire prostate gland regardless of the location of cancer. ${ }^{3}$ Moreover, with the perineal approach, the risk of infection is lower than that with conventional TRUS- $\mathrm{Bx}$, and it can also be performed in patients with anal anomalies. ${ }^{4-6,8}$ However, many studies have shown that the incidence of acute urinary retention (AUR) after TTMB is higher than that following TRUS-Bx..$^{4,6,7}$

Multiparametric magnetic resonance imaging (mpMRI) provides more detailed images of the prostate than those provided by standard MRI. 
mpMRI is expected to overcome the limitations of conventional biopsy methods for detecting prostate cancer. There are some reports that MRItargeted biopsy has similar or better results than systemic biopsy. Based on these reports, several guidelines recommend mpMRI before prostate biopsy. ${ }^{2,3}$ However, even if there are no suspicious lesions on mpMRI, cancers can be detected by TTMB. ${ }^{3}$ Furthermore, when administering focal therapy such as high-intensity focused ultrasound, the role of TTMB is reportedly essential for the confirmation of the target area.

Despite the importance of TTMB, clinicians should be aware of the complications following TTMB. Many studies about the complications after TTMB have been conducted, and AUR was reported as the most common complication after TTMB. ${ }^{10-15}$ Additionally, studies have assessed the factors predictive of AUR after TTMB. ${ }^{6,13,16}$ The factors identified include advanced age, large prostate volume, greater number of biopsies performed, higher severity of lower urinary tract symptoms before biopsy, and diabetes. ${ }^{13,16}$ However, previous studies were conducted on Western populations, and there has been no study involving Korean patients.

Asians have a smaller prostate volume than Westerners. ${ }^{17}$ Therefore, we hypothesized that the incidence of AUR and risk factors for AUR in Asians might be different from those in the Western populations. In this study, we investigated the complications after TTMB and the risk factors for AUR after TTMB in Korean patients.

\section{MATERIALS AND METHODS}

\section{Ethics Approval}

This study was approved by the Institutional Review Board of Samsung Medical Center (IRB No. 2021-01-074), and performed in accordance with the principles of the Declaration of Helsinki.

\section{Study Population}

We retrospectively reviewed 500 consecutive patients who underwent TTMB between May 2017 and July 2020 in a single tertiary center. We excluded 41 patients who were not removed urinary catheter day after TTMB by clinicians' decision. Finally, 459 patients were selected for this study.

The cases were categorized into 3 groups: (1) 255 cases in whom confirmatory biopsy after TRUS-Bx was performed for active surveillance (AS); (2) 176 cases with PSA elevation despite negative biopsy results on TRUS-Bx; (3) 28 cases that were biopsy-naive. Our institute performs AS only for very low-risk prostate cancers. TTMB was also performed in patients with increasing or persistently high PSA levels despite the prior negative TRUS-Bx results. TTMB was performed as the first biopsy in patients with rectal anomalies and in those susceptible to infectious disease.

\section{Biopsy Protocol}

TTMB was routinely performed under general anesthesia and guided by a 5-mm distance biopsy trial through a brachytherapy grid template. When the prostate volume was $<30 \mathrm{~mL}, 24$-core biopsy was performed. When the prostate volume exceeded $30 \mathrm{~mL}$, 36-core biopsy was performed under the "Ginsburg Protocol." Furthermore, if magnetic resonance imaging or ultrasonography results revealed suspicious lesions, a target biopsy core was added. Prostate biopsy cores were analyzed by a urologic pathologist.

Prophylactic IV antibiotics (fluoroquinolones) were injected before and one day after TTMB. Oral antibiotics were prescribed for 3 days after the patients were discharged. A urinary catheter 
was inserted after the TTMB procedure and was removed the day after TTMB. Clean intermittent catheterization (CIC) was performed when the patients failed to void after removal of the urinary catheter. A urinary catheter was reinserted if the patients failed to void after CIC. The urinary catheter was removed 1-2 days after reinsertion and hospitalization. In another case, the urinary catheter was removed 1 week later in the outpatient department.

\section{Definition of Complications}

We defined AUR as the need for reinsertion of the urinary catheter within 3 days after TTMB. Though retention was occurred after removal of urinary catheter, and it resolved by only 1 CIC, we did not define this event as AUR. Hematuria as a complication was defined as a patient visiting the emergency room or outpatient and readmission due to hematuria.

\section{Statistical Analysis}

The following descriptive statistics were used for continuous data: mean, standard deviation, and $95 \%$ confidence interval (CI) or median and interquartile range. Predictive factors for complications after TTMB were determined using a multivariate logistic regression model, and the odds ratio (OR) and 95\% CI were calculated. We chose cutoff values for each variable as mean values. Multivariate analyses were performed using factors found to be significant on univariate analysis. All statistical analyses were performed using IBM SPSS Statistics ver. 23.0 (IBM Co., Armonk, NY, USA). All p-values below 0.05 ( $p<0.05)$ were considered statistically significant.

\section{RESULTS}

\section{Patient Characteristics}

The patients' median (range) age at TTMB was 65 years (range, 37-82 years). The median PSA was 5.7 $\mathrm{ng} / \mathrm{mL}(0.03-42.7 \mathrm{ng} / \mathrm{mL})$. The wide variation in PSA levels was because some patients were diagnosed with prostate cancer after transurethral resection of the prostate or Holmium laser enucleation of the prostate. Some patients were on 5-alphareductase inhibitor for a long duration, and some underwent androgen deprivation therapy before TTMB. The median prostate volume was $38 \mathrm{~mL}$ (12.3-233 mL), and the median PSA density was 0.15 $\mathrm{ng} / \mathrm{mL}^{2}\left(0.00-0.96 \mathrm{ng} / \mathrm{mL}^{2}\right)$. The median number of total biopsy cores was 36 (24-40), and the median number of target biopsy cores was 2 (1-4). The median number of biopsies before TTMB was 1 (0-6). The indications for TTMB were categorized into 3 groups. Biopsy naive patients were 28 (6.1\%), patients with PSA elevation after negative TRUS-Bx was 176 (38.3\%) and patients with confirmative $B x$ after TRUS-Bx was 255 (55.6\%) (Table 1).

\section{Complications After TTMB}

Complications occurred in 95 patients (20.7\%). AUR occurred in 81 patients (17.7\%), and 14 patients (3.1\%) had hematuria. Clavian-Dindo grade IIIa complications occurred in only 1 patient with hematuria. The remaining 94 patients had ClavianDindo grade I complications (Table 2).

The patient with Clavian-Dindo grade IIIa complication had severe hematuria. Hence, the patient was not discharged, and the hemoglobin level decreased from 10.5 to $5.8 \mathrm{~g} / \mathrm{dL}$. Computed tomography angiography was performed, and contrast leakage was observed in the inferior vesical artery. The hematuria improved after immediate embolization. 
Table 1. Patient demographics and characteristics

\begin{tabular}{|c|c|}
\hline Variable & Value \\
\hline \multicolumn{2}{|l|}{ Age (yr) } \\
\hline Median (range) & $65(37-82)$ \\
\hline Mean \pm SD & $64.5 \pm 7.8$ \\
\hline \multicolumn{2}{|l|}{ PSA $(\mathrm{ng} / \mathrm{mL})$} \\
\hline Median (range) & $5.7(0.03-42.7)$ \\
\hline Mean \pm SD & $7.1 \pm 5.3$ \\
\hline \multicolumn{2}{|l|}{ Prostate volume $(\mathrm{mL})$} \\
\hline Median (range) & $38(12.3-233)$ \\
\hline Mean \pm SD & $43.0 \pm 21.5$ \\
\hline \multicolumn{2}{|l|}{ PSA density (ng/mL ${ }^{2}$ ) } \\
\hline Median (range) & $0.15(0-0.96)$ \\
\hline Mean \pm SD & $0.18 \pm 0.13$ \\
\hline \multicolumn{2}{|l|}{ No. of biopsy cores } \\
\hline Median (range) & $36(24-40)$ \\
\hline Mean \pm SD & $31.2 \pm 5.9$ \\
\hline \multicolumn{2}{|l|}{ No. of previous prostate biopsies } \\
\hline Median (range) & $1(0-6)$ \\
\hline Mean \pm SD & $1.1 \pm 0.6$ \\
\hline \multicolumn{2}{|l|}{ Additional target biopsy core } \\
\hline Yes, n (\%) & $131(28.5)$ \\
\hline \multicolumn{2}{|l|}{ No. of target biopsy cores } \\
\hline Median (range) & $2(1-4)$ \\
\hline Mean \pm SD & $2.1 \pm 0.6$ \\
\hline No, n (\%) & $328(71.5)$ \\
\hline \multicolumn{2}{|l|}{ TTMB indications } \\
\hline Initial TTMB, n (\%) & $28(6.1)$ \\
\hline Previous negative TRUS-Bx, $\mathrm{n}(\%)$ & $176(38.4)$ \\
\hline Confirmative biopsy after TRUS-Bx, n (\%) & $255(55.6)$ \\
\hline
\end{tabular}

SD: Standard deviation, PSA: prostate-specific antigen, TTMB: transperineal template-guided mapping biopsy, TRUS-Bx: Transrectal ultrasound-guided biopsy.

\section{Predictors of AUR After TTMB}

Logistic regression univariate analysis showed that age $\geq 65$ years (OR, 2.44; 95\% CI, 1.46-4.07; $\mathrm{p}=0.001$ ), PSA level $\geq 7 \mathrm{ng} / \mathrm{mL}$ (OR, 1.98; 95\% CI, $1.21-3.21 ; \mathrm{p}=0.006$ ), prostate volume $\geq 30 \mathrm{~mL}$ (OR, 8.44; 95\% CI, 3.34-21.38; $\mathrm{p}<0.001)$, and number of biopsy cores $\geq 30$ (OR, 6.26; 95\% CI, 3.28-11.93; $\mathrm{p}<0.001)$ were significant predictors associated with AUR following TTMB. On multivariate analysis, age $\geq 65$ years (OR, 2.44; $95 \% \mathrm{CI}, 1.42$ $4.17 ; \mathrm{p}=0.001$ ), prostate volume $\geq 30 \mathrm{~mL}$ (OR, 3.72; 95\% CI, 1.19-11.62; $\mathrm{p}<0.02)$, and total biopsy cores $\geq 30$ (OR, 2.89; 95\% CI, 1.29-6.43; p=0.01) were significant predictors associated with AUR following TTMB (Table 3).
Table 2. Complications after transperienal template-guided mapping biopsy

\begin{tabular}{cc}
\hline Complication & Value \\
\hline Acute urinary retention & $81(17.7)$ \\
Clavian-Dindo grade I & $81(17.7)$ \\
Hematuria & $14(3.1)$ \\
Clavian-Dindo grade I & $13(2.8)$ \\
Clavian-Dindo grade IIIa & $1(0.2)$ \\
No complications & $364(79.3)$ \\
\hline Values are presented as number (\%). \\
DISCUSSION
\end{tabular}

TRUS-Bx has played an important role in the detection of prostate cancer. ${ }^{1}$ However, there is a risk of infection after TRUS-Bx due to the anal approach, and infection after TRUS-Bx can progress to sepsis. ${ }^{3-8}$ TTMB can reduce the risk of urosepsis due to urinary tract infection (UTI), which is advantageous compared to TRUS-Bx, especially considering an increase in the number of antibiotic-resistant strains. ${ }^{18}$ In a study by Huang et al., $512 \%$ of the patients who underwent TRUS-Bx, developed UTI while the incidence was $2.2 \%$ in the TTMB group. The incidence of sepsis was $6.4 \%$ in the TRUS-Bx group and $0 \%$ in the TTMB group. In a study by Skouteris et al., ${ }^{6}$ UTI occurred in $4.2 \%$ of the patients who underwent TRUS-Bx and in only $0.79 \%$ of those who underwent TTMB. According to Berry et al., ${ }^{4}$ the TTMB group had a lower rate of hospitalization due to sepsis at $1.0 \%$ and compared to $1.4 \%$ in the TRUS-Bx group. However, TTMB also has some limitations. Several studies have reported that TTMB has a higher rate of AUR than TRUS-Bx. According to Skouteris et al., ${ }^{6}$ AUR did not occur in patients who underwent TRUS-Bx, but was seen in $7.9 \%$ of those who underwent TTMB.

In our study, a total of 459 patients who underwent TTMB were examined for post-TTMB complications. In terms of overall complications, our study showed that 95 of the 459 patients (20.7\%) experienced complications. According to Hara et al., ${ }^{19} 23$ out of 
Table 3. Logistical regression model to identify predictive factors for AUR after transperienal template-guided mapping biopsy

\begin{tabular}{|c|c|c|c|c|}
\hline \multirow{2}{*}{ Variable } & \multicolumn{2}{|c|}{ Univariable analysis } & \multicolumn{2}{|c|}{ Multivariable analysis } \\
\hline & OR $(95 \% \mathrm{CI})$ & p-value & OR $(95 \% \mathrm{CI})$ & p-value \\
\hline \multicolumn{5}{|l|}{ Age (yr) } \\
\hline$<65$ & & Reference & & Reference \\
\hline$\geq 65$ & $2.44(1.46-4.07)$ & 0.001 & $2.44(1.42-4.17)$ & 0.001 \\
\hline \multicolumn{5}{|l|}{ PSA (ng/mL) } \\
\hline$<7$ & & Reference & & Reference \\
\hline$\geq 7$ & $1.98(1.21-3.21)$ & 0.006 & $1.44(0.86-2.42)$ & 0.166 \\
\hline PSA density $\left(\mathrm{ng} / \mathrm{mL}^{2}\right)$ & $0.9(0.14-6.03)$ & 0.916 & & \\
\hline \multicolumn{5}{|l|}{ Prostate volume $(\mathrm{mL})$} \\
\hline$<30$ & & Reference & & Reference \\
\hline$\geq 30$ & $8.44(3.34-21.38)$ & $<0.001$ & $3.72(1.19-11.62)$ & 0.024 \\
\hline \multicolumn{5}{|l|}{ No. of biopsy cores } \\
\hline$<30$ & & Reference & & Reference \\
\hline$\geq 30$ & $6.26(3.28-11.93)$ & $<0.001$ & $2.89(1.29-6.43)$ & 0.01 \\
\hline
\end{tabular}

AUR: acute urinary retention, OR: odds ratio, CI: confidence interval, PSA: prostate-specific antigen.

126 patients (18.3\%) had post-TTMB complications. According to Takenaka et al., ${ }^{20} 19$ out of 100 patients (19\%) who underwent TTMB biopsy had complications. According to Tsivian et al., ${ }^{11} 16$ out of 84 patients (19\%) had complications after TTMB. Our study showed a similar overall rate of complications as that reported in several studies.

AUR was the most common complication and was seen in 81 patients (17.7\%) in our study. According to Kum et al., ${ }^{16} 31$ of the 243 patients (12.8\%) developed urinary retention. In a study by Buskirk et al., ${ }^{13} 11.5 \%$ of the patients developed AUR within 48 hours of the biopsy. According to Miah et al., ${ }^{15}$ among a total of 249 patients who underwent TTMB, 24\% experienced AUR. According to Skouteris et al., ${ }^{6} 7.9 \%$ of the total 379 patients, had post-TTMB AUR. Our study showed a somewhat higher rate of AUR than that reported in the aforementioned studies.

Hematuria was the second most common complication in our study. Hematuria was identified in 14 patients $(3.1 \%)$. In the previous studies, complications other than AUR have also been reported. According to Wang et al., ${ }^{21} 5$ out of 60 patients (8.3\%) had severe hematuria. According to Tsivian et al., ${ }^{11}$ prostatitis was $4 \%$. According to Pepe et al., ${ }^{10}$ total of 3,000 patients were separated to $12,18,24$ cores biopsy group, hematuria was found to be $8.1 \%, 9.7 \%$, and $10.4 \%$, respectively. Our study showed a relatively lower rate of complications except for AUR; however, ClavienDindo grade IIIa hematuria required intervention occurred in one patient.

In our study, age $\geq 65$ years, prostate volume $\geq$ $30 \mathrm{~mL}$ and total biopsy cores $\geq 30$ were identified as factors that had a statistically significant effect on AUR. The OR of age $\geq 65$ years was 2.44 (95\% CI, $1.42-4.17$; $=0.001$ ), for prostate volume $\geq 30 \mathrm{~mL}$ it was 3.72 (95\% CI, 1.19-11.62; $<<0.01$ ), and it was 2.89 (95\% CI, 1.29-6.43; p=0.01) for total biopsy cores $\geq$ 30. According to Buskirk et al., ${ }^{13}$ only the biopsy core numbers showed a statistically significant difference in multivariate analysis about urinary retention ( $\mathrm{p}=0.003$ ). According to Kum et al., ${ }^{16}$ there was a statistically significant difference in age between the urinary retention and nonretention groups (mean, 68.7 vs. 65.8 years, $\mathrm{p}=0.034$ ). The prostate volume also showed a statistically significant difference between the urinary retention and nonretention groups (mean, 75.4 vs. 57.2, $\mathrm{p}=0.0016$ ). Biopsy core also had a statistically significant difference between the urinary retention group and non retention group. (median, 35 biopsies vs. 32 biopsies, $\mathrm{p}=0.045$ ). According to 
Skouteris et al., ${ }^{6}$ results of the linear regression analysis about AUR factor showed statistically significant differences in age and prostate volume. Patients with prostate volume greater than $42 \mathrm{~mL}$ (median size) showed higher retention incidence for men with smaller prostates ( $13.4 \%$ vs. $2.7 \%$; OR, 5.7; 95\% CI, 2.1-15.1). Compared to cutoff value of prostate volume in this study $(30 \mathrm{~mL})$, other studies showed larger cutoff value of prostate volume. This result might be caused by smaller prostate volume in Korean population compared with Westerners.

There are some limitations to our study. First, this was a retrospective study with a relatively small sample size of 459 patients. Second, selection bias cannot be ruled out in a study conducted in a single tertiary center. Third, there is a possibility that the procedure was less consistent because TTMB was performed by several surgeons. Another study reported that a high International Prostate Symptom Score (IPSS) result before TTMB was a risk factor for AUR; however, we did not check the IPSS result before the procedure. ${ }^{7}$ Finally, according to the Ginsberg protocol, 24 biopsy cores were allocated if the prostate volume was less than $30 \mathrm{~mL}$, and 36 cores were allocated for prostate volumes larger than $30 \mathrm{~mL}$, which does not fully reflect the diversity of the volume state.

Despite these limitations, to the best of our knowledge, our study is the first to investigate the risk factors for AUR after TTMB in a Korean population. Compared to foreign data, our study showed that there was no significant difference in risk factors of AUR, but there were differences in specific values on risk factors. That might be caused by difference of prostate size between Westerners and Korean. Through these results, this study can serve as a reference for physicians to reduce the risk of AUR after TTMB in the Korean population.

\section{CONCLUSIONS}

AUR is the most common complication after TTMB. Age $\geq 65$ years, prostate volume $\geq 30 \mathrm{~mL}$, and number of biopsy cores $\geq 30$ were significant predictors of AUR following TTMB. Our results provide valuable information that may help clinicians to reduce the risk of complications following TTMB.

\section{CONFLICT OF INTEREST}

The authors claim no conflicts of interest.

\section{ORCID}

Si Hyun Sung, https://orcid.org/0000-0003-1777-6019

Chung Un Lee, https://orcid.org/0000-0002-3030-0405

Jae Hoon Chung, https://orcid.org/0000-0002-9729-3457

Wan Song, https://orcid.org/0000-0003-0971-1805

Minyong Kang, https://orcid.org/0000-0002-6966-8813

Hyun Hwan Sung, https://orcid.org/0000-0001-9845-3742

Byong Chang Jeong, https://orcid.org/0000-0002-5399-2184

Seong Il Seo, https://orcid.org/0000-0002-9792-7798

Seong Soo Jeon, https://orcid.org/0000-0002-3265-6261

Hyun Moo Lee, https://orcid.org/0000-0003-3969-4540

Hwang Gyun Jeon, https://orcid.org/0000-0002-5613-8389

\section{REFERENCES}

1. Litwin MS, Tan HJ. The diagnosis and treatment of prostate cancer: a review. JAMA 2017;317:2532-42.

2. Ahmed HU, El-Shater Bosaily A, Brown LC, Gabe R, Kaplan R, Parmar MK, et al. Diagnostic accuracy of multi-parametric MRI and TRUS biopsy in prostate cancer (PROMIS): a paired validating confirmatory study. Lancet 2017;389:815-22.

3. Lee CU, Sung SH, Jang CT, Kang M, Sung HH, Jeong $\mathrm{BC}$, et al. Cancer location in upgrading and detection after transperineal template-guided mapping biopsy for patients in active surveillance and nega- 
tive transrectal ultrasonography-guided prostate biopsy. Urol Int 2019;103:262-9.

4. Berry B, Parry MG, Sujenthiran A, Nossiter J, Cowling TE, Aggarwal A, et al. Comparison of complications after transrectal and transperineal prostate biopsy: a national population-based study. BJU Int 2020;126:97-103.

5. Huang GL, Kang CH, Lee WC, Chiang PH. Comparisons of cancer detection rate and complications between transrectal and transperineal prostate biopsy approaches - a single center preliminary study. BMC Urol 2019;19:101.

6. Skouteris VM, Crawford ED, Mouraviev V, Arangua P, Metsinis MP, Skouteris M, et al. Transrectal ultrasound-guided versus transperineal mapping prostate biopsy: complication comparison. Rev Urol 2018;20:19-25.

7. Loeb S, Vellekoop A, Ahmed HU, Catto J, Emberton M, Nam R, et al. Systematic review of complications of prostate biopsy. Eur Urol 2013;64:876-92.

8. Borghesi M, Ahmed H, Nam R, Schaeffer E, Schiavina $\mathrm{R}$, Taneja $\mathrm{S}$, et al. Complications after systematic, random, and image-guided prostate biopsy. Eur Urol 2017;71:353-65.

9. Rouviere O, Gelet A, Crouzet S, Chapelon JY. Prostate focused ultrasound focal therapy--imaging for the future. Nat Rev Clin Oncol 2012;9:721-7.

10. Pepe P, Aragona F. Morbidity after transperineal prostate biopsy in 3000 patients undergoing 12 vs 18 vs more than 24 needle cores. Urology 2013;81:1142-6.

11. Tsivian M, Abern MR, Qi P, Polascik TJ. Short-term functional outcomes and complications associated with transperineal template prostate mapping biopsy. Urology 2013;82:166-70.

12. Mai Z, Yan W, Zhou Y, Zhou Z, Chen J, Xiao Y, et al. Transperineal template-guided prostate biopsy: 10 years of experience. BJU Int 2016;117:424-9.

13. Buskirk SJ, Pinkstaff DM, Petrou SP, Wehle MJ, Broderick GA, Young PR, et al. Acute urinary retention after transperineal template-guided prostate biopsy. Int J Radiat Oncol Biol Phys 2004;59:1360-6.

14. Symons JL, Huo A, Yuen CL, Haynes AM, Matthews J, Sutherland RL, et al. Outcomes of transperineal template-guided prostate biopsy in 409 patients. BJU Int 2013;112:585-93.

15. Miah S, Eldred-Evans D, Simmons LAM, Shah TT, Kanthabalan A, Arya M, et al. Patient reported outcome measures for transperineal template prostate mapping biopsies in the PICTURE study. J Urol 2018;200:1235-40.

16. Kum F, Jones A, Nigam R. Factors influencing urinary retention after transperineal template biopsy of the prostate: outcomes from a regional cancer centre. World J Urol 2019;37:337-42.

17. Jeon HG, Yoo JH, Jeong BC, Seo SI, Jeon SS, Choi HY, et al. Comparative rates of upstaging and upgrading in Caucasian and Korean prostate cancer patients eligible for active surveillance. PLoS One 2017;12:e0186026.

18. Bader MS, Loeb M, Brooks AA. An update on the management of urinary tract infections in the era of antimicrobial resistance. Postgrad Med 2017;129:242-58.

19. Hara R, Jo Y, Fujii T, Kondo N, Yokoyoma T, Miyaji $\mathrm{Y}$, et al. Optimal approach for prostate cancer detection as initial biopsy: prospective randomized study comparing transperineal versus transrectal systematic 12-core biopsy. Urology 2008;71:191-5.

20. Takenaka A, Hara R, Ishimura T, Fujii T, Jo Y, Nagai A, et al. A prospective randomized comparison of diagnostic efficacy between transperineal and transrectal 12-core prostate biopsy. Prostate Cancer Prostatic Dis 2008;11:134-8.

21. Wang F, Ding X, Xu J, Xu Y, Zhou Y, Luan Y, et al. Complications of transperineal template-guided prostate mapping biopsy. Zhonghua Yi Xue Za Zhi 2019;99:428-31. 